\title{
Performance Evaluation System of Enterprise Knowledge Management Based on Balanced Scorecard
}

\author{
Mingkui Huo ${ }^{1} \& \mathrm{Li} \mathrm{Zhu}^{2}$ \\ ${ }^{1}$ School of Economy and Management, Changchun University of Science and Technology, Changchun, China \\ ${ }^{2}$ College of Information engineering, Changchun University of Science and Technology, Changchun, China \\ Correspondence: Mingkui Huo, School of Economy and Management, Changchun University of Science and \\ Technology, Changchun city, Weixing road 7989, China. E-mail: huomingkui@163.com
}

Received: June 29, 2014

Accepted: July 15, 2014

Online Published: July 23, 2014

doi:10.5430/bmr.v3n3p15

URL: http://dx.doi.org/10.5430/bmr.v3n3p15

\begin{abstract}
The performance evaluation system is the main method to measure the result of enterprise knowledge management. The text summarizes the functions of knowledge management at first, and then reviews the existing problems and defects in the studies of its kind, constructs the evaluation system of KM based on BSC, explains how to define the weights and operate the performance evaluation system in the end.
\end{abstract}

Keywords: Balanced scorecard, Knowledge management, Performance evaluation system

\section{Functions of Knowledge Management}

Since the beginning of the 21 st century, knowledge management (KM) has been widely discussed in academia and business circles. The view that knowledge is the key resource for enterprises to obtain competitive advantage has been universality accepted. Enterprise knowledge management is a process of coordination and management of knowledge acquisition, knowledge encoding, knowledge storage, knowledge transfer, knowledge sharing, knowledge application and knowledge innovation (Wang Zhongtuo, 2000). The purpose of knowledge management is promoting enterprise's ability of knowledge sharing to establish core capabilities (You Keqiang, 2003). Corresponding to the purpose, it is the basic functions of knowledge management. Based on the previous studies, the main functions can be concluded as followed, (Yang Changhui, 2007) (Zhang Xin, 2011) (Li Changling, 2006) (Zheng Jingli \& Si Youhe, 2003)

1) Arrange the internal information and make it in order

2) Collect external information and mine it

3) Establish corporate knowledge base; turn implicit knowledge into explicit one;

4) Make enterprise knowledge map to reduce the blindness and low efficiency of knowledge acquisition and knowledge transfer;

5) Provide technical support to knowledge sharing; improve the efficiency of knowledge sharing;

6) Foster knowledge-sharing atmosphere in enterprise and turn it into a learning organization;

7) Make strategies of knowledge management to serve the whole business development strategy;

8) Develop incentive and guarantee mechanism to motivate knowledge sharing in enterprise;

9) Knowledge application and knowledge innovation;

10) Evaluate knowledge management performance.

As there are various functions and the results are diverse, it is difficult to evaluate. An evaluation system should reflect enterprise knowledge management status. With it, we could find enterprises' existing problems and improvement directions by horizontal and vertical comparisons, to enhance its core competitiveness.

\section{Reviews of KM Performance Evaluation}

Domestic and foreign scholars have conducted a lot of studies on knowledge management performance evaluation, but, so far, they have been unable to form a unified understanding on its index system. 
Overseas, Quitas and his partners proposed the knowledge management performance evaluation system should consist of strategy polices of corporate knowledge development, knowledge acquisition and knowledge sharing, knowledge strategy implementation; business performance improvement resulted from knowledge management, testing and evaluation of related activities. Wiig believes that it should include monitoring and promoting intellectual activity, setting up and renewal the knowledge infrastructure, building knowledge assets, effective allocation of knowledge, knowledge application and knowledge learning. Andeersen creates the knowledge management assessment tool (KMAT), in which there are indexes in five dimensions including the sense of leadership, corporate culture, technology, assessment and changes of learning behaviors (Zhang Shaohui \& Ge Xinquan).

In China, scholars establish knowledge management performance evaluation systems from a couple of different perspectives. Searching the CNKI (Chinese National Knowledge Infrastructure) with the key words "Knowledge management and performance" in the period of 1990 to 2011, there are 149 relevant papers as total. After screening, there are 99 papers related to knowledge management performance evaluation, of which 7 are reviews, 71 are on its index systems and model designs, and the rest are relevant empirical studies on libraries, high-tech enterprises, firms, IT companies, consulting firms, universities and other organizations. After comparison, we find that their contents and methods are repeated in half of papers, so we just review several ones with prominent representative here.

Yan Guanghua and his partners build a three-index system in which stage targets are regarded as its first level indexes, including "immediate target", "medium-term target and "long-term target" (Yan Guanghua \& Li Jianwei, 2011). There is certain innovative in this idea. It facilitates controls of knowledge management performance and development. And it makes it easy to identify related influencing factors. But this operational sub-index system is not practical, for three stages exist simultaneously. With reviews from home and abroad, Zheng Jing li puts forward her enterprise knowledge management performance indicators system with six first-level indicators and 36 second-level indicators (Zheng Jingli \& Si Youhe, 2003). In that system, there are too much indicators, which are too difficult to measure and the correlations between levels are pretty weak. What is worse, it lacks indicators reflecting knowledge management infrastructure. Zhang Shaojie and his partners proposed a three-dimensional indicators evaluation system, in which indexes are on ontology evaluation, source evaluation, and strategic evaluation (Zhang Shaojie \& Wang Lianfen, 2004). It is rather comprehensive that it not only takes the process of knowledge management into account, but also considers the sources of knowledge and the ultimate goal of knowledge management. However, they did not construct detailed evaluation indexes, and there are other problems, such as lack of hierarchy, concept vagueness, index repeatability, imprecision, less quantifying and so on. Therefore, it is difficult to evaluate knowledge management performance with it. Taking the content, functions and objectives of enterprise knowledge management into account, Cao Xing and his partners set up an indicators system with six first-level indicators and 26 secondary indicators (Cao Xing, Chen Qi \& Peng Geng, 2006). It covers a wide range with a strong rationality, but it lacks indexes reflecting some special aspects such as finance, so it could be further improved. With the help of Balanced Scorecard, Zhou Zhiying and her partners construct a knowledge management performance evaluation system (Zhou Zhiying, TianCheng \& Wang Qifeng, 2009). It is very practical but not complete, for there is no index reflecting knowledge usage, knowledge sharing and other aspects, which is important to reflect the result of enterprise knowledge management performance.

On the whole, scholars construct knowledge management performance evaluation system based on organizational knowledge management framework, affecting factors, knowledge resources structure and knowledge processing activities types. For lack of comprehensive consideration, all of them are not perfect. They are neither comprehensive nor reasonable enough. Their data is unavailable and it is uneasy to imply. In addition, I believe that indicators of knowledge management performance evaluation and business performance evaluation should not be identical, but it occurred many times.

After reviews, this paper selected the most mature and practical tool, the Balanced Scorecard to design a more rational, comprehensive and practical evaluation system.

\section{KM Performance Evaluation System Based on the BSC}

In 1990s, the Balanced Scorecard (BSC) was putted forward by Capra and Norton. It is a multiple performance evaluation system that turns corporate strategies, decisions and tasks of internal departments into multiple, interrelated goals, and then breaks them down into a number of target indicators (Wang Jun \& Fan Zhiping, 2004). It overcomes the unicity and lag of evaluation only by finance. It evaluates value-creation activities in four aspects including financial, customer, internal business process, learning and growth. It not only focuses on the current corporate financial results, but also attaches the cause of long-term financial performance. So we can discover 
affecting factors of future performance, and create new value with more investments in customers, suppliers, employees, technology and innovation.

Knowledge management emphasizes organizational learning, knowledge sharing and innovation. It also focuses on process improvement to improve corporate service capacity, core competitiveness and earnings. The evaluation objects are knowledge and knowledge-based intangible assets, so the means should combined subjective with objective, quantify with qualitative. Setting up the evaluation system requires a tool which is able to evaluate both tangible and intangible benefits and overcome the defects of lag, one-sideness and low index representation. Therefore, it is reasonable to set up index system in four dimensions including financial, customer, internal process and learning and growth.

\section{The Design of Enterprise Knowledge Management Performance Evaluation System Based on the Balanced Scorecard}

The relationships between four levels of the Balanced Scorecard are progressive. This paper maps each knowledge management functions to Balanced Scorecard factors. For example, the establishment of learning organizations, the development of knowledge-sharing incentives and technical support to knowledge sharing corresponds to organizational capital, human capital, information capital of learning and growth dimension; the establishment of knowledge base, internal and external knowledge mining corresponds to internal business process dimension; knowledge mapping and knowledge sharing efficiency promotion corresponds to customer dimension, in which employees belong to enterprise's internal customers; knowledge application corresponds to innovation and financial dimensions.

\subsection{Indicators in Financial Dimensions}

Enterprise knowledge management performance is usually measured by return on assets, but indicators in financial dimensions are different. We use three indicators, cost reduction rate, net assets income growth rate and innovation achievement growth rate to reflect knowledge application and innovation.

\subsection{Indicators in Customer Dimension}

Indicators in customer dimension primarily refer to internal and external customer praise rate. The external customers praise rate could use indicators such as customer retention, market share growth, and brand image development to access. The internal customers praise rate could be measured by job satisfaction, per capita value creation improving rate and employees' abilities improvement which could be measured by leadership score, colleagues score and staff self-assessment score.

\subsection{Indicators in Internal Business Processes Dimension}

Indicators in this dimension reflect the coverage and depth of knowledge management, so we choose knowledge base coverage rate, knowledge base utilization rate, Knowledge inventory growth rate and learning activities growth rate of internal tacit knowledge transferring.

\subsection{Indicators in Learning and Growth Dimension}

The indicators of the Balanced Scorecard in learning and growth dimension mainly focus on what should be done to support business strategy realization, including organizational capital, human capital and information capital. When it comes to enterprise knowledge management, it refers to the construction of learning organization, the development of knowledge-sharing incentives and technical support to knowledge sharing. We select three indicators, flattening degree of organization, the development of learning team and the location and rank of Chief Knowledge Officer (CKO) to evaluate the development of learning organization. We also select the employees' satisfaction with knowledge innovation mechanism to evaluate the knowledge sharing incentives and knowledge management culture, and the proportion of knowledge management infrastructure investment in fixed assets to assess corporate technical supports. Abilities improvement which could be measured by leadership score, colleagues score and staff self-assessment score. 


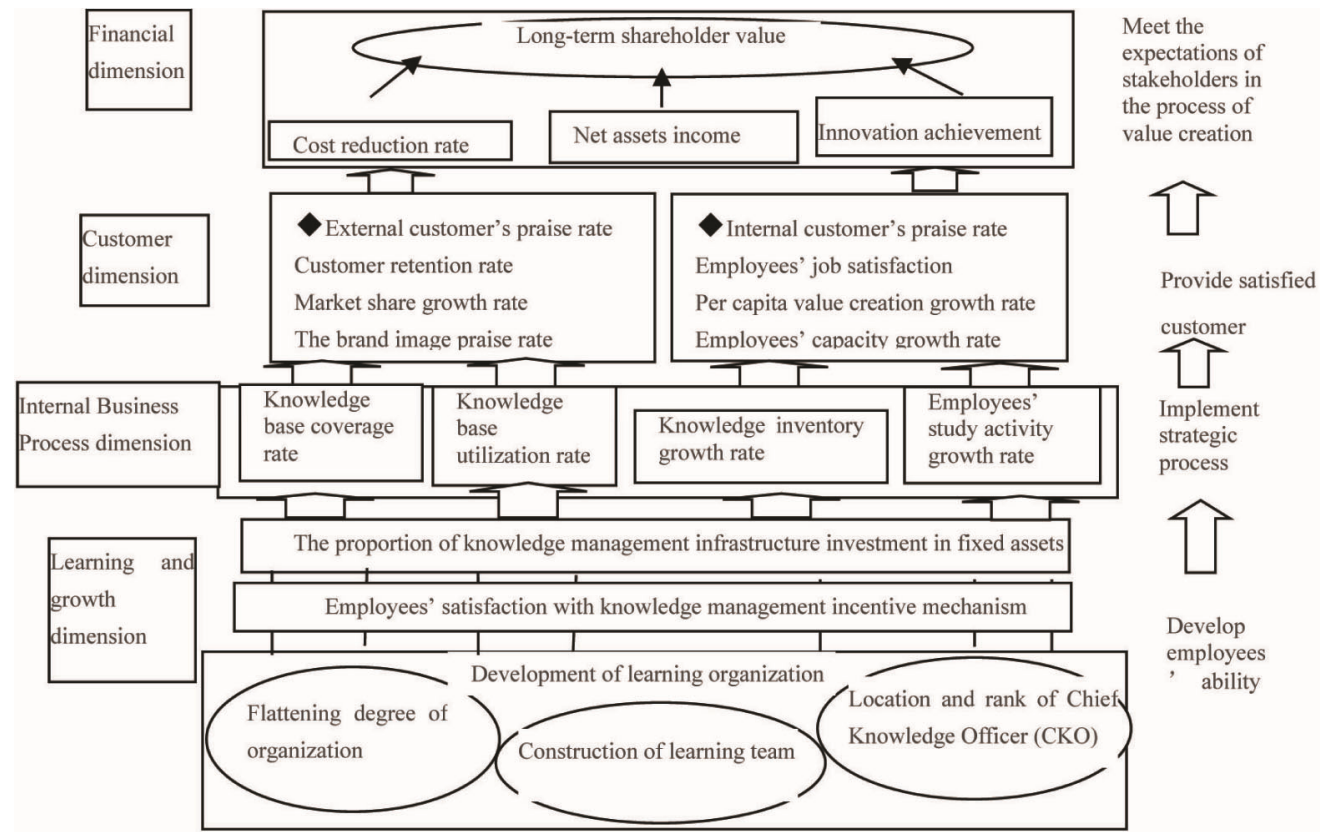

Figure 1. Enterprise knowledge management performance evaluation system based on the Balanced Scorecard Table 1. Enterprise knowledge management performance evaluation system based on the BSC

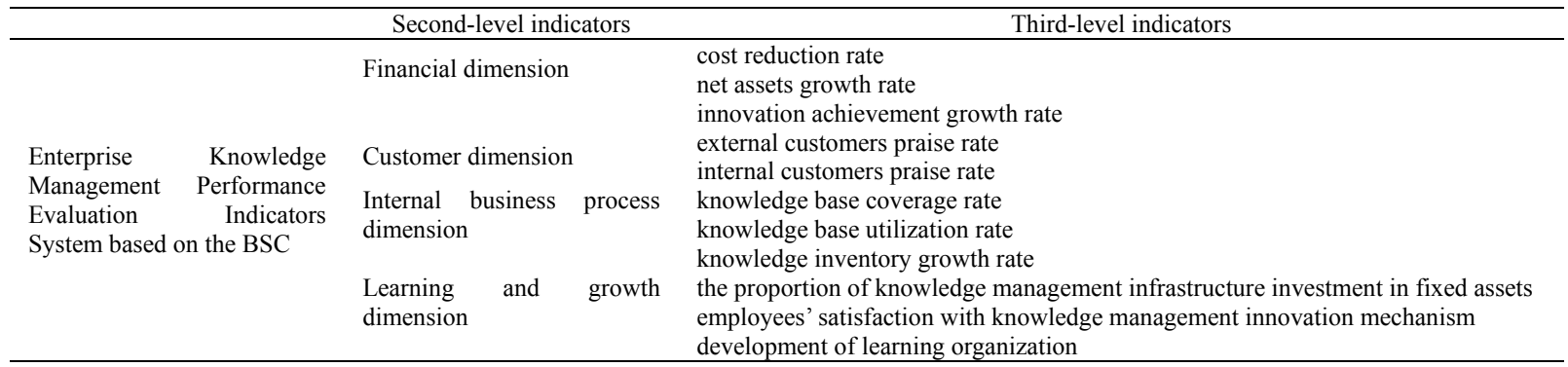

\section{Evaluation Model and Calculating}

This paper uses the Efficacy Coefficient method which is high mature and simple to explain the evaluation system.

\subsection{Index Setting}

The index system is divided into three layers.

The target layer $\mathrm{U}=\left(\mathrm{U}_{1}, \mathrm{U}_{2}, \mathrm{U}_{3}, \mathrm{U}_{4}\right)$;

First level indicators: $\mathrm{U}_{1}=\left(\mathrm{U}_{11}, \mathrm{U}_{12}, \mathrm{U}_{13}\right), \mathrm{U}_{2}=\left(\mathrm{U}_{21}, \mathrm{U}_{22}\right), \mathrm{U}_{3}=\left(\mathrm{U}_{31}, \mathrm{U}_{32}, \mathrm{U}_{33}, \mathrm{U}_{44}\right), \mathrm{U}_{4}=\left(\mathrm{U}_{41}, \mathrm{U}_{42}, \mathrm{U}_{43}\right)$;

\subsection{The Idea of Calculating}

The basic process of Efficacy Coefficient method is as follows:

Firstly, we turn indexes values dimensionless:

$$
U_{i}=\frac{U_{i}^{s}-U_{i}^{(c)}}{U_{i}^{(h)}-U_{i}^{(c)}} \times 40+60
$$

Ui is value of index i. $U_{i}^{s}$ is its actual value, while ${ }_{i}^{(h)}$ is its satisfaction value, which can be measured by cash or by evaluation after comparasion with benchmark enterprise, or it is just an ideal value; $U_{i}^{(c)}$ is the worst or unhallowed value. So values remain 60 to 100 .

\subsection{Weight Setting and Calculating Method}

Weight distribution is critical for performance evaluation. Whether it is reasonable directly determines the reliability and authenticity of evaluation. According to the Balanced Scorecard, the higher layer an index is in, the more emphasis it is on its income and effect, while the lower layer it is in, the more emphasis it is on basic work perfection. Therefore, we should set different weights in different stages. In early days, the weight of low-level indicators, such 
as $U_{3}$ and $U_{4}$, should be higher; when the workflow is stable, we distribute the weights in average; and finally when knowledge management strategy can be applied in a long term, the weight in high-level, such as $U_{1}$ and $U_{2}$, should be higher. The weight setting in the other secondary and tertiary target is also the case.

The specific weight setting is as followed: $\mathrm{P}_{\mathrm{i}}(\mathrm{i}=1,2,3,4)$ represents the weight of Ui, therefore the first level indicator weight is $\mathrm{P}=\left\{\mathrm{P}_{1}, \mathrm{P}_{2}, \mathrm{P}_{3}, \mathrm{P}_{4}\right\}, 0 \leq \mathrm{Pi} \leq 1$; the second level indicator weights are $\mathrm{P} 1=\left\{\mathrm{P}_{11}, \mathrm{P}_{12}, \mathrm{P}_{13}, \mathrm{P}_{14}\right), \mathrm{P} 2=$ $\left(\mathrm{P}_{21}, \mathrm{P}_{22}\right), \mathrm{P} 3=\left(\mathrm{P}_{31}, \mathrm{P}_{32}, \mathrm{P}_{33}, \mathrm{P}_{34}\right), \mathrm{P} 4=\left(\mathrm{P}_{41}, \mathrm{P}_{42}, \mathrm{P}_{43}\right)$, and the third level indicator weights $\mathrm{P}_{\mathrm{ij}}$ are just the case. Finally, we calculate the total value of $U$ with weighted geometric method, namely:

$$
U=\sqrt[\left(P_{1}+P_{2}+\ldots+P_{n}\right)]{U_{1}^{P_{1}} U_{2}^{P_{2}} \cdots U_{n}^{P_{n}}}
$$

The first level and second level indicators are calculated with that method. Finally, we assess performance by total score. If scoring 90 or more, it is perfect; between 80 and 89 is good; it is general between 70 and 79; it is poor between 60 and 69; below 60 is most poor. According to the result, enterprise is able to make horizontal and vertical contrast, so that knowledge management could be adjusted or improved.

\section{Conclusions}

Based on the Balanced Scorecard, we construct an enterprise knowledge management performance evaluation system. We calculate the scores with some practical and available quantitative indicators and the idea of benchmarking. This method can not only be used to evaluate the performance of enterprise knowledge management, but also to make horizontal contrast to find problems, so it has some theoretical and practical significance of enterprise knowledge management implementation.

\section{References}

Cao Xing, Chen Qi \& Peng Geng. (2006). Enterprise Knowledge Management Performance Evaluation Study. Science and Technology Management.

Li Changling. (2006). Fuzzy Evaluation of Knowledge Management Performance. Information Science.

Wang Jun \& Fan Zhiping. (2004). A Comprehensive Evaluation Method of Organizational Knowledge Management Performance. Journal of Management Engineering.

Wang Zhongtuo. (2000). Knowledge System Engineering: New Discipline of Knowledge Management. Journal of Dalian University of Technology.

You Keqiang. (2003). Knowledge Management and Innovation. Tsinghua University Press.

Yang Changhui. (2007). The Research on the Goal Framework of Knowledge Management and its Influencing Factors. Technology \& Management Research.

Yan Guanghua \& Li Jianwei. (2001). Knowledge Management Performance Evaluation Study. NanKai Business Review.

Zhang Shaohui \& Ge Xinquan. Fuzzy Evaluation Model and Matrix Analysis of Enterprise Knowledge Management Performance.

Zhang Shaojie \& Wang Lianfen. (2004). Factor Analysis and Index System of Enterprise Knowledge Management Performance Evaluation. Information Science.

Zhang Xin. (2011). Review of Enterprise Knowledge Management. China Science and Technology Forum.

Zheng Jingli \& Si Youhe. (2001). Enterprise Knowledge Management Evaluation Index System Research. Economic System Reform.

Zhou Zhiying, TianCheng \& Wang Qifeng. (2009). The Study Of Enterprise Knowledge Management Performance Evaluation based on BSC and fuzzy evaluation. Library and Information Service. 